\title{
History and Physical Exam
}

National Cancer Institute

\section{Source}

National Cancer Institute. History and Physical Exam. NCI Thesaurus. Code C135566.

A record of the medical history and physical examination findings for a patient collected at the time of admission. 